\title{
The Royal Observatory, Greenwich
}

\section{Annual Visitation}

$\mathrm{T}$ HE annual visitation by the Board of Visitors of the Royal Observatory, Greenwich, presided over by the president of the Royal Society, took place on June 3, when the Astronomer Royal, Dr. H. Spencer Jones, presented his report for the year ending April 30, 1939. The report includes the following sections: instruments and apparatus; astronomical observations; time service; Nautical Almanac Office ; magnetic observations; meteorological observations.

Transit Circle Observations. The programme for laying the foundations of a system of right ascensions with the new reversible transit circle has been continued. Since its inception in 1937, 4,333 transits have been observed; 1,501 collimations and 1,975 levels were taken in the course of these observations. Intensive observations have traced the main cause of the diurnal changes of collimation to small tem. perature differences between the micrometer screw and the eyepiece box, amounting sometimes to $2^{\circ} \mathrm{F}$. Similarly, changes of level appear to be due largely to temperature differences between the two piers of the instrument, arising from unequal shielding in the case of any wind that is not due north or south. The adopted collimation and level errors are now derived from smoothed curves drawn through the values given by the observations; drift in the clock error has thereby been practically eliminated.

The Airy transit circle has been devoted chiefly to the observation of the sun, moon and planets. The Greenwich observations of the sun from 1750 , combined with observations made at other observatories and used in conjunction with observations of the moon and Venus and of the transits of Mercury, form the data for investigating the changes in the rate of rotation of the earth. The results are being communicated to the Royal Astronomical Society.

Cookson Zenith Telescope. 283 plates were taken for the determination of latitude variation. A definitive discussion of the observations for the years 1911-1936 is in the course of publication.

28-inch Refractor. The new comparison-image micrometer continues to prove superior to the filar micrometer, especially for the measurement of the closer double stars.

Thompson Equatorial, 26-inch Refractor. 1,238 plates for parallax determinations were secured and 38 parallaxes entirely completed.

Yapp 36-inch Reflector. With the slitless spectrograph the colour-temperature programme was com. pleted in September. The discussion and detailed results for 250 stars will shortly be in the press.

The slit spectrograph has now taken the place on the telescope of the slitless spectrograph. In addition to experimental work, a provisional programme has been started, consisting of one-prism exposures, for the ultra-violet, on yellow and red giant spectroscopic binaries, to see whether a hot companion can be detected in this region of the spectrum even though its lines are smothered at longer wave-lengths.

Photoheliograph and Spectrohelioscope. A daily series of photographs (begun in 1874) has been secured, in collaboration with the Cape Observatory and Kodaikanal Observatory, for the measurement of sunspot positions and areas. There are indications that the peak of the maximum phase of the 11-year cycle has been passed. Eleven very large spot groups appeared during the year ; in seven cases, a magnetic storm occurred within a few days of the respective times of central meridian passage. Examination of the sun's disk is made in hydrogen light $(H \alpha)$ to detect the sudden occurrence of bright eruptions which show a relationship with ionospheric irruptions. Observations also include photometric measures of hydrogen disk markings and of prominences at the sun's edge.

Time Service. The official time services have been regularly maintained. The principle of employing a number of clocks as a 'mean clock' has been further extended and has proved of great assistance. The astronomical determination of clock error was made on 114 nights with a reversible transit instrument. The quartz crystal clock constructed at the National Physical Laboratory has been installed. The performance of the Westminster clock ("Big Ben") showed that, while on 16 days the error was greater than ls., on 188 days the error did not exceed $0.5 \mathrm{~s}$. During the year, 4,170 chronometers and watches were received and 3,662 issued. The stock of chronometers and watches now held at the Observatory is being decentralized.

Nautical Almanac Office. During the year, the work of the Office has been mainly devoted to the routine calculation and publication of the Nautical Almanac, the Abridged Nautical Almanac, the Air Almanac and the Astronomical Navigation Tables. A working unit is being organized to deal with special investigations that frequently arise. A special supplement to the Almanac for 1940 will give data required in the design of instruments and their final adjustment. The paths of the ionospheric and corpuscular eclipses of October 1, 1940, have been published.

Magnetic Observations. A set of La Cour instruments for quick-run recording ( $3 \mathrm{~mm}$. to the minute) has been added to the equipment at Abinger. The mean values of the magnetic elements for 1938 are :

Declination West, $11^{\circ} 1 \cdot 4^{\prime}$

Horizontal Intensity, $0 \cdot 18522$ gauss

Vertical Intensity, $0 \cdot 43050$ gauss

Inclination, $66^{\circ} 432^{\prime}$

Magnetic activity during the year was considerable, five great storms and twenty-three small storms being recorded. The disturbance of the earth's magnetic field at Abinger by artificial fields due to electric traction is stated to have become increasingly serious as the electrification of the Southern Railway has extended. Disturbance $(5 \gamma$ to $10 \gamma)$ is continuous throughout the working hours and ceases only during the interval lh. $-5 \mathrm{~h}$. civil time. The complexity of the total effect makes identification of the separate origins ordinarily impossible. The installation of the La Cour quick-run magnetograph has provided evidence, however, that significant effects occur coinciding with the movements of trains in the neighbourhood of Guildford as well as of Dorking. In the last few months, field investigations of the effect of electric traction have been made near 
Chichester, at Itchenor and at Selsey. At the latter site, seven miles from the nearest point of the railway, disturbance was quite distinct, although seldom amounting to $1 \gamma$ in vertical intensity.

Staff. The post of chief assistant, rendered vacant by the appointment in the previous year of Mr. W. M. H. Greaves to the post of Astronomer Royal for Scotland, has been filled by Dr. H. R. Hulme, fellow of Caius College, Cambridge.

In concluding his report, the Astronomer Royal states the concern he feels regarding the steady deterioration of observing conditions at Greenwich. Long-exposure photography is no longer possible owing to the increasing brightness of the sky at night. As a result of atmospheric pollution by smoke, programmes of work which used to be successfully undertaken at Greenwich are now impracticable. The actual definition of stellar images has also suffered. In addition, there are various forms of fouling, the two most serious being the deposit of grit and the destruction of aluminized surfaces. The pivots and Y's of the new reversible transit, although carefully protected, already show appreciable scoring. There is a permanent risk of damage by grit to the objectives of all the Greenwich telescopes. "If the Royal Observatory is to continue to make important contributions to astronomy as it has done for the past 264 years, and to provide an efficient time service of high accuracy, it is essential," the report continues, "that it should be removed to a site where astronomical conditions are favourable. The present wasteful efforts to secure observations under increasingly bad conditions, and the restrictions of programmes that are necessitated by such conditions, would thereby be avoided."

Finally, as regards the Abinger magnetic station, the general level of artificial disturbance is now such as to render all observations difficult, and when the disturbances are at their worst the observations lose much of their value. The removal of the magnetic observatory to another site has therefore become a necessity. The choice of a new site for such work in the south of England appears limited to a few regions in Devonshire.

The report concludes with the statement that proposals for the removal of the whole of the astronomical work from Greenwich and of the magnetic work from Abinger to new and more favourable sites are under consideration.

\section{Historical Survey of Mycology*}

$\mathrm{U}$ NLIKE the treatment of flowering plants, so far as Linnæus was concerned, the subject of mycology had scarcely begun before his time. It is true that many fungi were mentioned by the classical writers but most of the interest in them was derived solely from their gastronomic effects. Their origin puzzled certain writers who regarded them as the result of the fermentation of the earth; truffles, on the other hand, were supposed to be due to the action of lightning. Herbals were based on classical authority : in 1593 Cesalpino wrote suggesting that fungi were not plants and were spontaneously generated.

Towards the end of the sixteenth century, Porta recognized the spores of such dark-spored forms as Coprinus and possibly of the mycetozoan Fuligo septica; but he thought they were seeds.

The arrival of the microscope was necessary before any progress in mycology could be made; but even then progress was slow. Hooke, in his "Micrographia", devoted two sections to fungi, and his description of the rose blight, Phragmidium, was accompanied by an illustration which is the first known diagram of a microscopic fungus. Hooke's views on fungi were thus expressed : "First, that Moulds and Mushrooms require no seminal property, but the former may be produc'd at any time from any kind of putrifying Animal, or Vegetable Substance, as flesh, etc., kept moist and warm.... Next, that as Mushrooms may be generated without seed, so does it not appear that they have any such thing as seed in any part of them ; for having considered several kinds of them, I could never find any thing in them that I could with any probability guess to be the seed of it, so that it does not yet appear (that $I$ know of) that Mushrooms may be generated from a seed, but they rather seem to depend merely upon a convenient constitution of

- From the Presidential Address delivered to the Linnean Society of London, by Dr. J. Ramsbottom, O.B.E., on May 24. the matter out of which they are made, and a concurrence of either natural or artificial heat." In spite of accumulation of knowledge, similar ideas on the fungi extended over the following century and a half.

Malpighi, writing during 1675-79, described certain fungi in which he interpreted the sporangiophore as an inflorescence and the spores as florets. Thus, Hooke favoured the spontaneous origin of fungi, whereas Malpighi favoured the origin from seed.

Apart from Leeuwenhoek's observations on yeast in 1690, the next step was the recognition of the mycelium or spawn of mushrooms. Tournefort, in 1707, gave the first account of mushroom-growing, and described the mycelium. Still further speculative theories of the origin of fungi appeared, until in 1729 P. A. Micheli published what may be considered as the first real step in our knowledge of the fungi. $\mathrm{He}$ made many morphological observations of first importance, but was actually still looking for flowers and seeds in the fungi examined. He also proved that what we now know as spores gave rise to new fungi similar to the plants from which they were taken. But in spite of Micheli's work, most botanists gave up the fungi as hopeless, and even Linnæus in his "Philosophia Botanica" of 1751 wrote: "The order of Fungi is still Chaos, a scandal to art, no botanist knowing what is a species and what a variety."

Misled by the observations of Baron O. F. Munckhausen, Linnæus, in the twelfth edition of his "Systema Naturæ" described a genus "Chaos" with six species; the species "C. Fungorum" showed the metamorphosis of zoophytes from vegetables into animals and vice versa. "C. Ustilago" is the modern Ustilago which infects grains of cereals, grasses and certain florets. "C. infusorium" contained various Protozoa. The genus was rightly named, and thus did Linnæus sadly come to grief.

John Ellis, who had a long correspondence with 